\title{
Mental health policy adoption as a seminal event: A response
} to recent commentaries

\section{Gordon C Shen*}

*Correspondence to: Gordon C Shen, Email: gordon.shen@yale.edu Copyright: ( 2015 by Kerman University of Medical Sciences

Citation: Shen GC. Mental health policy adoption as a seminal event: A response to recent commentaries. Int J Health Policy Manag. 2015;4(7):493-494. doi:10.15171/ijhpm.2015.90

Received: 8 April 2015, Accepted: 22 April 2015, ePublished: 24 April 2015

$\mathrm{T}$ The leadership for mental health is not commensurate with the burden of mental, neurological, and substance (MNS) use disorders nationally or internationally. This is a sentiment I share with Prof. Jenkins (1) and Ms. Lee (2). With that said, I would like to make two clarifications about my study and two concomitant acknowledgements about its limitations (3). First, I conceptualized national mental health policy adoption as an isolated event. Policy adoption is one - albeit pivotal-node embedded in a ratification process. Second, I focused solely on external actors' influence on policy adoption. Politics and policy are intertwined, and there are certainly actors situated inside, as well as outside, each country who are engaged with mental health policy-making, but they were not addressed by my study. I will elaborate on what I set out to do before giving pointed responses to their comments. My global and historical account of the presence of mental health on national policy-making agendas is the result of harmonizing theory, quantitative methods, and different empirical contexts. The theoretical position I assumed was derived from the world polity theory (4). The world polity provides a set of cultural norms and guidance for each member country in dealing with the MNS burden prevalent in their population. The prediction is that mental health policy adoption, a trigger to mental health reform, would happen successively across countries due to their individual relationships with the World Health Organization (WHO) ("coercive isomorphism"), with their region and with one another ("mimetic isomorphism"). I chose discrete event history analysis to examine policy diffusion across countries in the WHO Mental Health Atlas and other secondary datasets. And I found trace evidence in support of these mechanisms that cut across geopolitical contexts and bind countries together.

Prof. Jenkins and Ms. Lee have pointed out some of the limitations of my study, and offered interesting points of departure for me and other researchers. Studies designed using quantitative and/or qualitative methods can reveal different facets of mental health reform, which I concur with both Prof. Jenkins and Ms. Lee, and I used the former. For instance, "normative isomorphism", a third potential mechanism of convergence in mental health policy adoption, was not accounted for in my study. Professional, research, civil society networks that span multiple countries can and do proffer tacit, scholarly, and practitioner knowledge to influence policy $(5,6)$. The role these intermediaries play in national policy fora and their power relations are largely absent from multi-national databases, including the WHO Mental Health Atlas. The WHO and the Gulbenkian Global Mental Health Platform did jointly field a survey to the epistemic community involved with deinstitutionalization (7). Two of the five key principles respondents identified for deinstitutionalization is that political support at the highest and broadest level is crucial, and that policy change happens during certain windows of opportunity. Stakeholder and network analyses are just as important as quantitative analyses in shedding light on whether and how the target that the WHO stipulated for $80 \%$ of its member countries to develop or update their mental health policy in line with international and regional human rights instruments can be achieved by 2020 (8). The results of my study can only speak to the rate at which policy development occurred leading up to 2011. An update of my study using data from the 2014 WHO Mental Health Atlas, currently underway, could show whether adoption rates continued at the same rate beyond 2011. Finally, my study is an assessment done on the country level of analysis, but future studies would be well-served by tailoring my approach to elucidate the science behind the formulation and reformulation of evidence-based mental health policy subnationally.

Mental health policies, unlike plans or legislations, are broad statements declaring decision-makers' commitment to address the burden of MNS disorders prevalent among their citizens. Policy commitments would need to be coupled with and be reinforced by funding flows and governance structures to be fulfilled. In answering to Lee about the need to examine policy effectiveness in addition to policy adoption, Prof. Snowden and I would refer readers to another published study where I compared changes different policy adoption groups made in population-scaled psychiatric bed rates over the course of a decade (9). We found that countries that are late-adopters of mental health policy are more likely to reduce psychiatric beds in mental hospitals and other biomedical settings than innovators, whereas they are less likely to reduce psychiatric beds in general hospitals than non-adopters. We thus inferred that late adopters are motivated to implement deinstitutionalization for technical efficiency reasons rather than social legitimacy reasons. The two quantitative studies are related in their theoretical rationale and empirical contexts examined. Policy commitments have a lagged impact on the process indicators Prof. Snowden and I scrutinized. 
Policy's impact on outcome indicators such as morbidity and mortality take even longer to be realized.

Sea changes in global agreements after my study period are causes of optimism, but it remains to be seen whether the same changes will to be reflected on the national level. Most notably, the WHO's Mental Health Action Plan (2013-20) was adopted by 194 member states during the 66th World Health Assembly. Mental health also has political support from bilateral funding agencies such as Grand Challenges Canada. However, reporting against set targets remains nascent given how recent these international commitments were made to mental health. To elaborate on the point made by Prof. Jenkins, mental healthcare and substance abuse control are both subsumed under the code 12191 for "Medical services" in the Organisation for Economic Co-operation and Development (OECD) Creditor Reporting System. Systematic, cross-national collection of data, including aid data would ultimately improve the way mental health is treated as a policy issue.

National mental health policy adoption is a seminal, focusing event which changes the status quo of treatment and prevention for MNS disorders. Ideally, it sets into motion the reform of mental health system in an iterative and recursive manner. IJHPM readers would hopefully find the vectors coming out of the exchange between me, Prof. Jenkins, and Ms. Lee inspiring for their future research.

\section{Ethical issues}

Not applicable.

Competing interests

Author declares that he has no competing interests.
Author's contribution

GCS is the single author of the manuscript.

References

1. Jenkins R. Making psychiatric epidemiology useful: the contribution of epidemiology to government policy. Acta Psychiatrica Scandinavica 2001; 103: 2-14. doi: 10.1111/j.16000447.2001.00004.x

2. De Silva MJ, Lee L, Fuhr DC, Rathod S, Chisholm D, Schellenberg J, et al. Estimating the coverage of mental health programmes: a systematic review. Int J Epidemiol 2014; 43: 341-53. doi: 10.1093/ije/dyt191

3. Shen GC. Cross-national diffusion of mental health policy. Int J Health Policy Manag 2014; 3: 269-82. doi: 10.15171/ ijhpm.2014.96

4. Meyer JW, Boli J, Thomas GM, Ramirez FO. World society and the nation-state. Am J Sociol 1997; 103: 144-81. doi: 10.1086/231174

5. Finnemore M, Sikkink K. International norm dynamics and political change. International Organization 1998; 52: 887-917. doi: $10.1162 / 002081898550789$

6. Mackenzie J. Global mental health from a policy perspective: a context analysis. London: Overseas Development Institute; 2014

7. World Health Organization (WHO). Innovation in Deinstitutionalization: A WHO Expert Survey. Geneva, Switzerland: WHO; 2014.

8. World Health Organization (WHO). Mental Health Action Plan 2013-2020. Geneva, Switzerland: WHO; 2013.

9. Shen GC, Snowden LR. Institutionalization of deinstitutionalization: a cross-national analysis of governance gap and mental health reform. Int J Mental Health Syst 2014; 8: 47. doi: 10.1186/1752-4458-8-47 